\title{
Special issue on shared and cooperative control
}

\author{
F. Flemisch ${ }^{1,2} \cdot$ D. Abbink ${ }^{3} \cdot$ M. Itoh ${ }^{4} \cdot$ M.-P. Pacaux-Lemoine ${ }^{5}$
}

Published online: 24 October 2019

(c) Springer-Verlag London Ltd., part of Springer Nature 2019

Keywords Human-machine systems $\cdot$ Human-machine cooperation $\cdot$ Joint action $\cdot$ Shared control

This special issue bridges a gap between the worlds of manually controlled human machine systems and so called autonomous systems, where the machine is in control alone. In contrast to autonomous systems, with Shared Control and its extension Cooperative Control both the human(s) and the machine(s) are involved in the control. This can be either simultaneously and/or alternating in time, and with different proportions of share. Shared and cooperative control paradigms have already been developed in parallel in many domains, e.g. aviation, in ground vehicles and robotics, in industry, crisis management and the military. In addition to the applied research and development that has taken place, an increasing part of the community has worked on the theory and concepts of human machine cooperation with its instantiations in shared and cooperative control. With increasing capabilities and connectivity of machines, e.g. in automated driving and flight, or in Industry 4.0, the

F. Flemisch

f.flemisch@iaw.rwth-aachen.de

D. Abbink

d.a.abbink@tudelft.nl

M. Itoh

itoh.makoto.ge@u.tsukuba.ac.jp

M.-P. Pacaux-Lemoine

marie-pierre.lemoine@uphf.fr

1 Institute of Industrial Engineering and Ergonomics, RWTH Aachen University, Aachen, Germany

2 Fraunhofer Institute for Communication, Information Processing and Ergonomics, Wachtberg, Germany

3 Department of Cognitive Robotics, Faculty of 3mE, Delft University of Technology, Delft, The Netherlands

4 University of Tsukuba, Tsukuba, Ibaraki 305-8573, Japan

5 Laboratoire d'Automatique et de Mécanique Industrielles et Humaines, Université Polytechnique Hauts de France, Valenciennes, France challenge and chance of sharing and cooperating on guidance and control will become even more important.

An introductory paper by the guest editors "Joining the blunt and the pointy end of the spear: towards a common framework of joint action, human-machine cooperation, cooperative guidance and control, shared-, traded- and supervisory control" looks deep into the history of co-operation between humans and their tools with the goal of unifying the plethora of related concepts and definitions that have been proposed in recent years. Definitions are provided to relate these concepts and to sketch a unifying framework of shared and cooperative control that sees the different concepts as different perspectives or foci on a common design space of shared intentionality, control and cooperation between humans and machines. The relationship between shared control and human-machine cooperation is compared to the relationship between the sharp, pointy tip and the (blunt) shaft of a spear. Shared control is where cooperation comes sharply into effect at the control layer, but to be truly effective it should be supported by cooperation in all layers beyond the operational layer, i.e. in the tactical and strategic layers. A fourth layer addresses the meta-communication about the cooperation and supports the other three layers in a traversal way.

The paper "A critique of the SAE conditional driving automation definition, and analyses of options for improvement" by Inagaki and Sheridan starts with criticism of the state of art of automation levels in the automotive domain, as defined for example by the U.S. Society of Automotive Engineers. Since conditional automation suffers from being crisply defined in terms of shared and cooperative control, it contains risks for drivers and manufacturers. This paper provides a method to derive an optimal design for the Request to Intervene (RTI) and proves that automation level 3 coupled with the optimal RTI should never be simply called "conditional driving automation". 
The next paper by Pacaux-Lemoine and Flemisch explores the "Layers of shared and cooperative control, assistance and automation", with a meta-model of automation and its historic development over the years. The meta models based on insight from the $\mathrm{H}$ (orse)-methaphor and human-machine cooperation principles are presented and combined in order to propose a framework and criteria to design safe, efficient, ecological and attractive human machine systems.

The paper by Baltzer et al. introduces the concept "Towards an interaction pattern language for human machine cooperation and cooperative movement", where shared and cooperative control is structured as an interaction pattern similar to the design pattern in architecture and software design. An essential element of these patterns are image schemes, which relate to fundamental psychological schemes in our understanding of and interaction with the world, here applied to human machine cooperation in a driving context.

The paper by Altendorf et al. on "Utility assessment in automated driving for cooperative human-machine systems" instantiates game theory and utility functions in a cooperative driving automation, and tests the approach with a driving simulator experiment in an highway overtaking situation.

The contribution by Wessel et al. "Self-determined nudging: a system concept for human-machine interaction" introduces a new way of interaction in cooperative human machine systems. Nudging is a concept from behavioural economics that works with subtle interactions. In contrast to traditional nudging, in self-determined nudging the human authorises the machine at an earlier stage, to influence his or her behaviour at a later stage.

The paper by Wada addresses one of the most fundamental challenges with automation, skill degradation. They postulate that instead of losing skills with inappropriate automation, a "Simultaneous achievement of driver assistance and skill development in shared and cooperative controls" is possible, and they demonstrate this with a haptic shared control parking system.

The paper "Principles of transparency for autonomous vehicles: first results of an experiment with an augmented reality human machine interface" by Pokam et al. describes how design principles form the base for a successful shared and cooperative control and present a study using augmented reality and cooperative automation in a driving simulator.

In their paper "Driver-vehicle cooperation: a hierarchical cooperative control architecture for automated driving systems" Guo et al. describe the importance of hierarchy in cooperation, which should also be reflected in the architecture of the human machine system, which is then also reflected in the technical architecture of the machine. They demonstrate this with the case of cooperatively controlled driving.

In "Effects of risk-predictive haptic guidance in one-pedal driving mode" Saito and Raksincharoensak investigate how elderly drivers can be assisted in risk-predictive driving via three shared and cooperative guidance control systems, and how elderly drivers can effectively take evasive action to ensure safety in the response-time critical scenario of a pedestrian initiating a road-crossing from a driver's blind area.

"A theoretical framework for designing human-centered automotive automation systems" by Muslim and Itoh deals with the design and evaluation of adaptive shared control system as compared to an adaptive traded control system. The objective is to study authority and strategies for control transitions according to levels and types of automation, as well as their impact on the safety and performance of the human-machine system.

"Shared control architectures for vehicle steering" by Ghasemi et al. deals with the design and use of system models of Input Mixing and Haptic Shared Control. The objective is to support smooth transitions of authority and control between human and automation, by helping the human driver in the building of a driving assistance model, enabling monitoring and the prediction of the behaviour of the automation.

Finally, in "Action prediction with the Jordan model of human intention: a contribution to cooperative control", Schneemann and Diederichs propose that the success of cooperative or shared control depends to a large extent on a common goal and hence on the successful prediction of the cooperators' goals. Recognizing the partner's intentions so as to predict their action is a fundamental basis for cooperation. A new model of prediction of the human intention is described and evaluated.

Publisher's Note Springer Nature remains neutral with regard to jurisdictional claims in published maps and institutional affiliations. 